\title{
Road Quality and Ghats Complexity analysis using Android sensors
}

\author{
Prof. V. P. Tonde ${ }^{1}$,Amol Jadhav ${ }^{2}$, Shreyas Shinde ${ }^{3}$, Ashish Dhoka ${ }^{4}$, Sandeep Bablade ${ }^{5}$ \\ Professor, Department of Information Technology, Sinhgad Institute of Technology Lonavala, India ${ }^{1}$ \\ BE Final Year, Department of Information Technology, Sinhgad Institute of Technology Lonavala, India ${ }^{2,3,4,5}$
}

\begin{abstract}
Importance of the road infrastructure for the society could be the same as importance of blood vessels for human`s. Road surface quality should be monitored and repaired on a regular basis. It is very difficult to design a optimal system which gathers the road condition data and processes it. Participatory sensing approach can be mostly used for such data collection. The paper is describes a mobile sensing system (android application) for road irregularity detection using Android OS based smart phone sensors. Selected data processing algorithms are discussed and their evaluation presented with true positive rate as high as $90 \%$ using real world data. The optimal parameters for the algorithms are determined as well as recommendations for their application.Continuously keeping track on road and traffic conditions in a city is a problem widely studied. Many methods have available towards addressing this problem. But this methods proposed require dedicated hardware such as GPS devices and accelerometers in vehicles or cameras on roadside and near traffic signals. All such proposed are unaffordable tothe common man regarding of monetary cost and human effort required. We extend a prior study to improve the algorithm based on using accelerometer, GPS and magnetometer sensor readings for trafficand road conditions detection. We are specifically made research in identifying braking events - frequent braking indicates congested traffic conditions - and bumps on the roads to characterize the type of road.
\end{abstract}

Keywords: Mobile sensing; Android phones; Bumps; Accelerometers; GPS; Algorithms.

\section{INTRODUCTION}

Roads have become a very important factor of our day to day life. Travelling gives us speed which we certainly cannot acquire by walking. Proper roads will lead to less time consumption for travelling and makes it less vulnerable of accidents. When road is made available to people for use after the completion of its construction it goes under various anomalies like continuous rolling of heavy vehicles, weather conditions like heavy rains, storms, snow etc. This will adversely show its effect on quality of driving. With the availability of information regarding the road conditions, road users can be cautious about or avoid the bad roads. It is desirable to have a mechanism for detecting the condition of roads and get them repaired as soon as possible. As a result, working on monitoring road conditions has gained significant attention in recent time. Dangerous road surface conditions are major distractions for safe and comfortable transportation. Both drivers and road maintainers are interested in fixing them as soon as possible. However, these conditions have to be identified first.

One approach to road damage detection is to use human reports to central authorities. While it has the highest accuracy,assuming that people are fair, it also has the most human interaction and is not comprehensive. Statistical analysis can be used to estimate damage probabilities of road segments based on their usage intensity. Integration of vibration and vehicle counting sensors in the pavement are used for statistical data collection. Surface analysis methods using Ground Penetrating Radar (GPR) have been developed and commercial products do exist. Unfortunately, this technology is using expensive equipment and therefore limits its accessibility.

Copyright to IJARCCE

DOI 10.17148/IJARCCE.2015.4324
As an alternative, participatory sensing has the potential to increase the collected data resolution and scope. The simplest method might be to collect photos of road damage and hazards taken by the participants and to upload them to a central server. However, this requires strong participation and interaction from the users as well as manual image analysis. We believe that an automated approach for detecting potholes with little or no human interaction is more promising. This would ensure more comprehensive survey data with less errors caused by human factors than generated by mere enthusiasm of the participants.

To create a successful road surface monitoring system accepted by wide user community, it is important to make it attractive for the users - to provide added value without a significant process overhead.

Therefore we envision our system as a service, which is added as a layer to existing navigation systems, such as Google Maps, which use real-time traffic information, collected by participatory sensing approach. Although contemporary smart-phones have high processing power and considerable memory, the detection system is recommended to avoid resource-intensive detection methods and to preserve initial user interface responsiveness. Conceptual image of our approach is shown in the figure 1 below.

To realize the final goal, in the first phase, which is the scope of this paper, it is important to find out and understand the relationship between acceleration data from smartphones and actual road surface condition. Furthermore, it is also important to understand to what extent different variables such as speed, vehicle type and

Copyight to IJARCCE

(1) 
smartphone setting may have on the mentioned relationship.

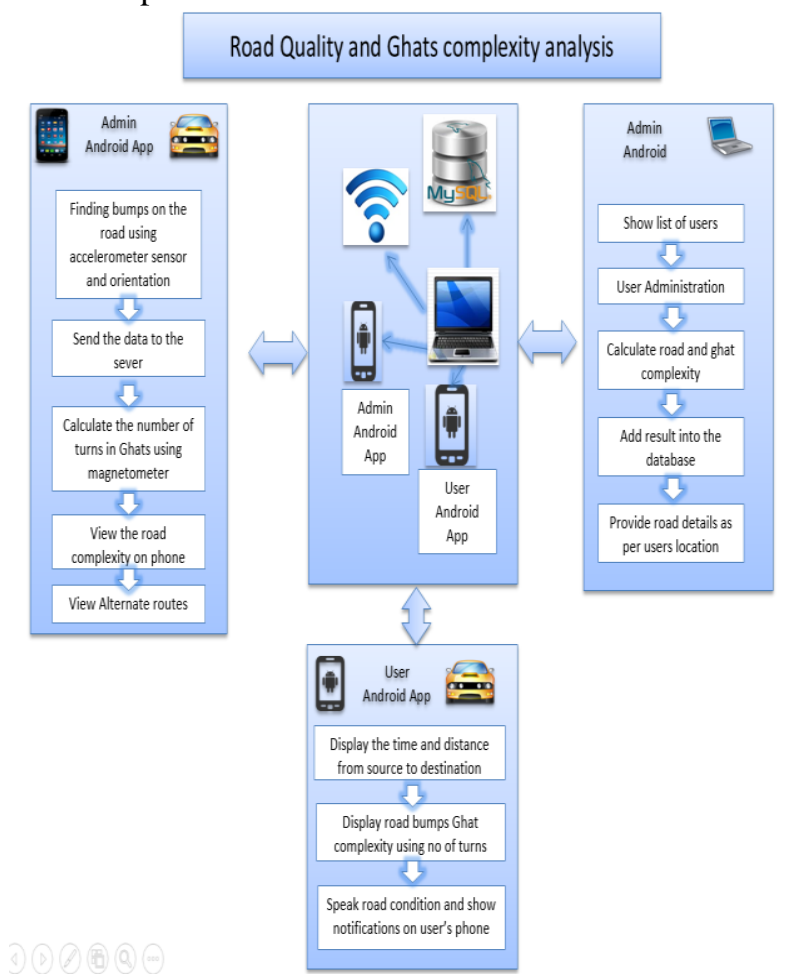

Fig.1 Conceptual image of continuous road condition monitoring system

\section{METHODOLOGY}

Assuming that different road surface conditions cause vehicles to vibrate differently, therefore by placing smartphones that come with acceleration sensors, the variation of the vibration is believed to be captured. In our experiment, we place a smartphoneon vehicles. We then drive each vehicle with normal driving condition along many roads that have different surface conditions. Different approximately fixed drivingspeeds are also performed on four selected short sections $(0.6$ to $1 \mathrm{~km}$ in length). The four short sections consist ofdifferent surface conditions such as good, fair, poor, and badrespectively. Sensors on the smartphones record acceleration and GPS data. To simplify our experiment, orientation of the smartphones is fixed. Thus, we assume that the acceleration coordinates of the vehicle and smartphones are the same.

After obtaining the data, analysis, which includes matching, sectioning of acceleration data into small files, and then each file is converted to analyse in frequency domain, has been carried out.

\section{III.DATA COLLECTION}

Equipment required to carry out the experiment are an Android phone with Accelerometer sensor, A server to process the data and a vehicle to collect the data.A smartphone is pre-installed with an application called "Advanced Road Traveller". This is an application that can collect data from almost all of the sensors available on the handsets. For this experiment and studies, only acceleration data $(\mathrm{x}, \mathrm{y}, \mathrm{z})$ from accelerometer; location data (longitude, latitude, speed...) from GPS are needed. Data recording is done at an interval of 0.01 second or at a frequency rate of $100 \mathrm{~Hz}$. Calibration is done for all 4 devices with Application by setting the devices in different orientations to collect data; after that data from the devices is compared against each other for validation purpose.

Data from smartphones is uploaded into a desk top computer and converted to excel spreadsheets. The spreadsheets are carefully checked manually to select only road sections that have complete data sets.

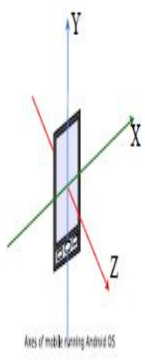

Phone Axes

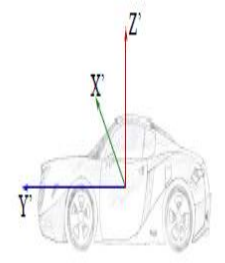

Vehicle Axes

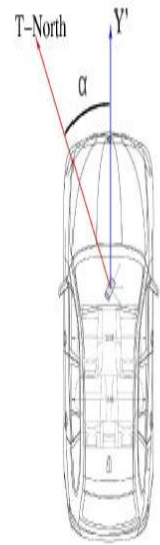

Direction of Motion
Fig. 2 Axes Synchronization

\section{IV.ALGORITHM}

\section{Road Bump Detection Logic-}

Based on the experiment result, road bump detection logic is designed as follows:

Condition 1: Both of the Y-axis or running direction and $\mathrm{Z}$-axis or vertical direction, $50[\mathrm{~ms}]$

Standard deviation is large.

Condition 2: These sections are appeared with wheelbase time.

Here, each variable is defined as follows. A recording order number is defined ' $i$ '. An acceleration data are defined X(i), Y(i), Z(i) for each axis. For Y-axis or running direction and $\mathrm{Z}$-axis or vertical direction, 50[ms] standard deviation is defined SDy(i), SDz(i). For the condition 1, simultaneity index is defined SDyz(i), and it is calculated by equation 1 .

$\operatorname{SDyz}(\mathbf{i})=\operatorname{SDy}(\mathbf{i}) * \operatorname{SDz}(\mathbf{i})$ (equation 1)

Cycle number of wheelbase time is defined Nw. For the condition 2, Bump Index is defined

$\operatorname{Byz}(i)$, and it is calculated by equation 2 .

$\operatorname{Byz}(\mathbf{i})=\operatorname{SDyz}(\mathbf{i}) * \operatorname{SDyz}(\mathbf{i}+\mathbf{N w})$ (equation 2)

Nwis related with vehicle speed. Vehicle speed is defined $\mathrm{V}[\mathrm{m} / \mathrm{s}]$. Wheelbase is definedLw[m].Recording cycle is defined $\mathrm{H}[\mathrm{Hz}]$. Nwis calculated by equation 3 .

$\mathbf{N w}=(\mathbf{L w} / \mathrm{V}) * \mathbf{H}$ (equation 3) 
Standard Deviation

$$
\sigma=\sqrt{\frac{\sum(x-\bar{x})^{2}}{N}}
$$

where

$$
\sigma=\text { the standarddeviation }
$$$$
x=\text { each value in the population }
$$

$$
\begin{aligned}
& \bar{x}=\text { the mean of the values } \\
& N=\text { the number of values (the population) } \\
& \text { Fig. } 3 \text { Standard Deviation }
\end{aligned}
$$

\section{Location Based Distance Calculation}

This uses the 'haversine' formula to calculate the circle distance between two points - that is, the shortest distance over the earth's surface - giving an 'as-the-crow-flies' distance between the points.

Haversine formula:

$\mathrm{a}=\sin ^{2}(\Delta \varphi / 2)+\cos \left(\varphi_{1}\right) \cdot \cos \left(\varphi_{2}\right) \cdot \sin ^{2}(\Delta \lambda / 2)$

$\mathrm{c}=2 \cdot \operatorname{atan} 2(\sqrt{\mathrm{a}}, \sqrt{ }(1-\mathrm{a}))$

$\mathrm{d}=\mathrm{R} . \mathrm{c}$

Where,

$\Phi$ is latitude, $\lambda$ is longitude, $\mathrm{R}$ is earth's radius(means radius $=6,371 \mathrm{~km}$ ) note that angles need to be in radians to pass to trig functions!

\section{ANALYSIS RESULT}

An experiment condition is as follows. For GPS positioning, smartphone should stay under the sky. Therefore smartphone is put on the vehicle dashboard in front of passenger's seat. In this experiment, any mounting equipment is not used, just only used a thin rubber sheet for an anti slipping. The Samsung Galaxy Grand 2 is used for the smartphone. The install direction of Phone is display side is upside and the home button is vehicle back side. It means that $\mathrm{X}$-axis of the accelerometer is width direction, $\mathrm{Y}$-axis is running direction, $\mathrm{Z}$-axis is vertical direction. A recording cycle is $100[\mathrm{~Hz}]$.

It means that, when the vehicle is driven at $60[\mathrm{~km} / \mathrm{h}]$, travel distance is $0.17[\mathrm{~m}]$ at one recording cycle. GPS recording cycle is about every $1[\mathrm{sec}]$. For emulate a bump step, round wooden rod which has $24[\mathrm{~mm}]$ diameter and $900[\mathrm{~mm}]$ long, is put on the asphalt road. And the vehicle goes over this rod, and the tire of passenger side is climbing over this rod. The vehicle speed is $30[\mathrm{~km} / \mathrm{h}]$. In this driving, a driver felt the bump step, but not felt a danger. It means that this bump step is not so large. The vehicle is running clockwise 5 laps on the square course which has $620[\mathrm{~m}]$ long for one lap. The 1 [sec] standard deviation of $\mathrm{Z}$-axis acceleration which is the vertical direction is drawn with circle diameter. The round wooden rod is located at arrowed position on the right bottom side of Fig.3, but it is difficult to find the bump step form this figure.

Copyright to IJARCCE

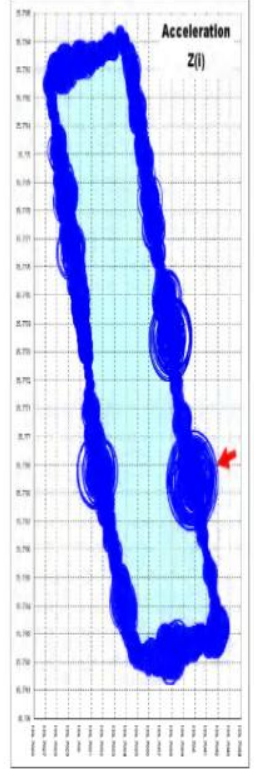

50[ms] Standard Deviation SDz(i)

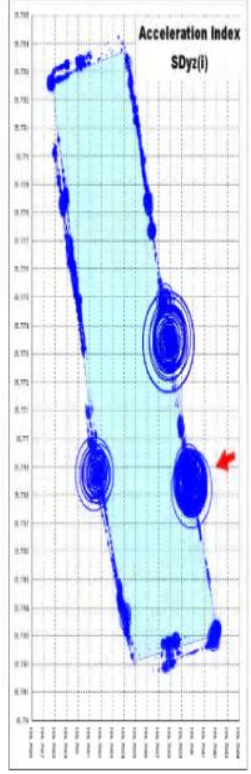

Simultaneity Index SDyz(i)

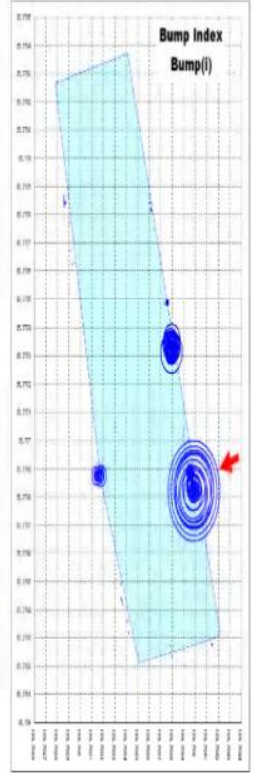

Bump Index Byz(i)
Fig. 4 Analysis Graphs

\section{CONCLUSION AND FUTURE WORK}

This paper explores features and relationships between acceleration data, collected bysmartphones, and road roughness condition. With the assumption that rough estimation of road surface condition from smartphones would be helpful enough for road management and planning, provided that the approach is very low cost, easy to operate and can be implemented frequently.

An experiment is carried out to obtain data. The data is checked and matched with referenced data. The matched data then cut into sections representing many 100 meter road sections. Analysis is carried out in frequency domain to calculate magnitudes of acceleration data. An adopted group of road condition indexes have been proposed. From the analysis, it has been found that acceleration data from smartphones has linear relationship with road roughness condition. However, the significant of relationship depends on speed in which it is considerably significant when speed is less than 60kph. Furthermore the relationship also partly depends on vehicle type and device. Based on the condition indexes, similar tendency of the classification of the sum of magnitudes of acceleration vibration is observed.

Although we can conclude that a simple model may be sufficient to estimate road roughness condition from acceleration data obtain by smartphones, there are still many issues that have to be dealt with in our future works to make the approach practically applicable. Main focus in the future works includes:

- Detail studies on the features and the relationship of the acceleration data and road roughness condition. Realistic smartphones setting, not fixed coordinate, will also be considered.

- Explore approaches to estimate road surface condition from many anonymous road/smartphone users, who agree 
to participate. Plenty of data for many different road sections would enable us to build models to simulate and estimate road roughness condition and soundness of road infrastructure as a whole.

\section{REFERENCES}

[1] GunjanChunj, DivyaBansal, SanjeevSofat; Road Condition Detetion using Smartphone Sensors: A Survey; In: International Journal of Electronic and Electrical Engineering,June 2009

[2] Ravi Bhoraskar, NagamanojVankadhara, Bhaskaran Raman, PurushottamKulkarni; Wolverine: Traffic and Road Condition Estimation using Smartphone Sensors;In: IEEE February 2012.

[3] FatihOrhan, P. ErhanEren,; Road Hazard Detection and Sharing with Multimodal Sensor Analysis on Smartphones; In: Seventh International Conference on Next Generation Mobile Apps, September 2013

[4] Yagi, Koichi; Extensional Smartphone Probe for Road bump detection

[5] Mohan, P., Padmanabhan, V.N., Ramjee, R. (2008) Nericell: Rich Monitoring of Road and Traffic Condition using Mobile Smartphones. Proceedings of the 6th ACM Conferenceon Embedded Network Sensor Systems, 323-336.

[6] Perttunen, M., Mazhelis, O., Cong, F., Kauppila, M., Leppänen, T., Kantola, J., Collin J., Pirttikangas, S., Haverinen, J., Ristaniemi, T. (2011) Distributed road surface condition monitoring using mobile phones. Ubiquitous Intelligence and Computing, 64-78.

[7] Viengnam DOUANGPHACHANH, Hiroyuki ONEYAMA;A Study on the Use of Smartphones for Road Roughness Condition Estimation. 